\title{
Efecto (¿o no?) de la inclusión de naturaleza de la ciencia en una secuencia para el aprendizaje y la aceptación de la teoría de la evolución
}

\author{
Lilian Moreno ${ }^{1, \mathrm{a}}$, Katherine Zuñiga ${ }^{1, \mathrm{~b}}$, Hernán Cofrée, ${ }^{2, \mathrm{c}}$, Cristian Merino ${ }^{2, \mathrm{~d}}$ \\ ${ }^{1}$ Colegio Carampangue, Talagante, Chile. \\ ${ }^{2}$ Pontificia Universidad Católica de Valparaíso, Valparaíso, Chile. \\ aalilianmoreno.a@gmail.com, bkathyz18@gmail.com, chernan.cofre@pucv.cl, \\ dcristian.merino@pucu.cl
}

[Recibido: 27 Noviembre 2017. Revisado: 17 Mayo 2018. Aceptado: 12 Junio 2018]

\begin{abstract}
Resumen: La presente investigación tuvo por objetivo implementar una secuencia de enseñanza y aprendizaje (SEA de aquí en adelante), donde se trabajaron dos grandes actividades en cinco sesiones, que abarcaron las cuatro etapas del ciclo de aprendizaje (exploración, introducción, estructuración y aplicación). En esta SEA, se incorporaron algunos aspectos de Naturaleza de la Ciencia ( $\mathrm{NdC}$ de aquí en adelante) como estrategia de enseñanza, sintonizando así con resultados de otras investigaciones que sugieren que su inclusión puede mejorar la aceptación y comprensión de la evolución. La investigación tuvo un diseño cuasi-experimental, donde hubo dos tratamientos de trabajo en tres cursos $\left(\mathrm{n}_{\text {total }}=48\right)$, en dos de los cursos se trabajó con $\mathrm{NdC}$ y un tercer curso fue el grupo control que trabajó sin NdC. Se realizaron en los tres cursos cuestionarios previo y posterior a la implementación de la SEA, para conocer la visión de Ciencia (Cuestionario VNOS-D+), aceptación de la Evolución (Cuestionario MATE), y el grado de conocimiento de Evolución de los estudiantes (instrumento propio de la investigación). Para el análisis de los resultados se realizó el test no paramétrico de comparaciones de medias relacionadas Wilcoxon Signed- Rank, para lograr visualizar con exactitud la relevancia de los resultados. Estos mostraron que el curso que trabajó con SEA y donde $\mathrm{NdC}$ se explicaba de forma explícita, mejoró de forma significativa su comprensión de $\mathrm{NdC}$ y la aceptación de la evolución, en comparación a los otros dos cursos. Sin embargo, todos los cursos mejoraron el conocimiento sobre evolución, siendo más significativo el aumento del curso que no tuvo $\mathrm{NdC}$ implementado en la SEA. Estos resultados sugieren que al utilizar aspectos de $\mathrm{NdC}$ para enseñar evolución, no solo se comprende de mejor forma, si no que los estudiantes tienen una mayor aceptación del contenido en comparación a cuando se trabaja evolución sin $\mathrm{NdC}$.
\end{abstract}

Palabras clave: Secuencia de Enseñanza y Aprendizaje (SEA), Ciencias Naturales, Evolución, Naturaleza de la Ciencia (NdC).

Effect (or not) of including the nature of science in a sequence for learning and acceptance of the theory of evolution

\begin{abstract}
The objective of this research was to implement a teaching-learning sequence (TLS), where two major activities were carried out in five sessions, covering the four stages of the learning cycle (exploration, introduction, structuring and application). In this TLS some aspects of Nature of Science as a teaching strategy were incorporated, attuned to results of other researches that suggest that their inclusion can improve the acceptance and understanding of evolution. The research had a quasi-experimental design where there were two treatments of work in three groups $(\mathrm{n}=48)$, in two of the groups was worked with NoS and a third groupe was the control that worked without NoS. They were made in the three prior and subsequent to the implementation of the TLS questionnaires courses to meet the vision of Science (Questionnaire vnos-D), acceptance of evolution (Questionnaire MATE), and degree of knowledge development of students (instrument of research). For analysis of the results the nonparametric Wilcoxon mean comparisons related Signed- Rank, in order to visualize accurately the relevance of the results was performed, which showed that the course worked with TLS and where we explained so explicitly significantly improved their understanding of NoS and the acceptance of evolution, compared to the other two courses. However, all the courses improved the knowledge about evolution, being more significant the increase of the course that did not have NoS implemented in the TLS. These results suggest that when using aspects of NoS to teach evolution, it is not only better understood, but also students accepted more the content than when working evolution without NoS.
\end{abstract}

Keywords: Teaching-learning sequence (TLS), Science, Evolution, Nature of Science (NoS). 


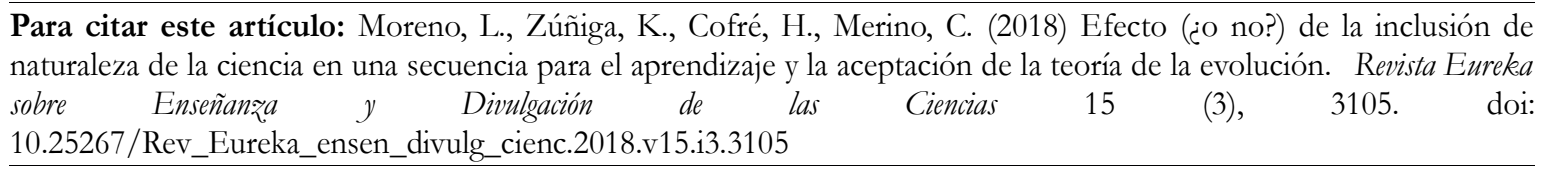

\section{Introducción}

La idea central que da sentido a la Biología moderna es la Evolución. Theodosius Dobzhansky lo resumió en su famosa declaración: «Nada en Biología tiene sentido, salvo en el contexto de la Evolución» (Dobzhansky 1973).

Sin embargo, diversas investigaciones (Cofré, Vergara, Santibáñez y Jiménez 2013; Cofré, Santibañez, Jiménez, Spotorno, Carmona, Navarrete y Vergara 2017; Barberá 2009, Puig y Jiménez-Aleixandre 2009; Cañal 2009; González-Galli y Meinardi 2015, 2017) han mostrado que muchos estudiantes poseen una visión poco adecuada de cómo se ha generado el conocimiento científico que subyace a la teoría de la evolución. Además, se ha descrito que los estudiantes presentan una serie de preconcepciones relacionadas con la incapacidad de comprensión del mecanismo a nivel poblacional y no en base a las necesidades de cada individuo en su relación directa con el ecosistema.

Los principales obstáculos descritos en el proceso de enseñanza y aprendizaje de la evolución, en estudiantes que inician la adolescencia, tienen que ver con las preconcepciones o concepciones alternativas (Gonzalez-Galli y Meinardi 2015). En relación a ellas, numerosas investigaciones convergen en señalar que parecen estar ampliamente difundidas y comparten los siguientes rasgos: son, en ciertos aspectos, semejantes a la teoría Lamarkiana de la evolución (Jiménez-Aleixandre 1991); suponen que el cambio evolutivo es consecuencia del cambio individual (Bardapurkar 2008); se basan en la noción de necesidad (Cofré et al. 2013 Cofré, Cuevas y Becerra 2017); involucran la noción de herencia de los caracteres adquiridos (Banet y Ayuso 2003); suponen que el cambio evolutivo obedece a fines predeterminados o teleología (Kampourakis y Zogza 2008); están relacionadas con la visión de naturaleza de la ciencia (NdC) de los estudiantes (Lombrozo, Thanukos y Weisberg 2008). Específicamente en este último tema, numerosos estudios han descrito concepciones específicas sobre el estatus del conocimiento evolutivo que mantienen los estudiantes como por ejemplo: a) la evolución es solo una teoría; b) la evolución no tiene evidencia empírica que la apoye; c) la investigación en evolución no puede seguir el método científico porque no se pueden hacer experimentos, entre otras (Cofré et al. 2017; Dagher y BouJaoude 2005; Glaze y Goldston 2015; Kim y Nehm 2010). En base a esto, se ha sugerido que la enseñanza de la evolución incluya explícitamente el trabajo de algunas de estas concepciones sobre el conocimiento evolutivo, en especial, el concepto de teoría y el reconocimiento de las evidencias empíricas del mecanismo de selección natural (Bayer y Luberda 2016; Cofré et al. 2017). En este trabajo se muestran resultados sobre la generación de una secuencia de enseñanza y aprendizaje (SEA) de la evolución, que incluye el tratamiento explícito de NdC y su implementación en estudiantes de octavo año de educación primaria en Chile.

\section{Marco de referencia}

En Chile, el currículo nacional de ciencias (Ministerio de Educación [Mineduc] 1998, 2009) incluye el tema de evolución desde octavo año básico (13 años, $2^{\circ}$ ESO). Sin embargo, en cursos anteriores ( $5^{\circ}$ básico, 10 años) comienza la revisión de conceptos relacionados como: adaptación, población, especie, biodiversidad, entre otros, para terminar en octavo básico, con la revisión de los orígenes de la vida y la historia evolutiva de los diferentes grupos de organismos a través del registro fósil (Mineduc 2009). No obstante, la mayor cobertura y profundidad se alcanza en la enseñanza media, donde se revisan temas de variabilidad genética, herencia, se- 
lección natural, evolución humana, entre otros (Mineduc 2009; Cofré et al. 2013). En este documento oficial además, se establece la importancia de la comprensión de NdC en los estudiantes, señalando «este sector tiene como propósito que los estudiantes adquieran una comprensión del mundo natural y tecnológico, y que desarrollen habilidades de pensamiento distintivas del quehacer científico» (Mineduc 2012).

Con objeto de confeccionar la SEA y poder incluir aspectos de $\mathrm{NdC}$, se revisaron diferentes autores que sugieren cómo enseñarla en el aula (McComas 1998; Clough 2003; Lederman, Abd-el-Khalick, Bell y Schwartz 2002). Pero para los fines de este trabajo, se adoptó la propuesta de Lederman y colaboradores en la construcción y desarrollo de la SEA planificada para el contenido de evolución, ya que tras analizar cada una de sus ideas y principios, es esta última la que nos permite trabajar de forma más concreta con nuestros estudiantes aspectos de $\mathrm{NdC}$, ya que reconoce un grupo de aspectos concretos de la $\mathrm{NdC}$ que son más cercanos a lo que los profesores y profesoras pueden trabajar en el aula (véase Kampourakis 2016 para una discusión sobre el aporte de las distintas formas de enseñar $\mathrm{NdC}$ ). Los rasgos de la $\mathrm{NdC}$ descritos por Lederman et al. (2002) son los siguientes: a) provisional o sujeto a cambios; b) fundamentado empíricamente; c) parcialmente subjetivo o guiado por la teoría; d) en parte producto de la inferencia humana; e) en parte producto de la imaginación y la creatividad del investigador; f) teorías científicas y leyes son diferentes constructos, y g) empapado social y culturalmente.

Para lograr la comprensión del contenido de evolución con aspectos de la $\mathrm{NdC}$, se desarrolló una SEA, que se define como un documento de planificación correspondiente a un tema o un contenido curricular concreto (Couso, 2012). En rigor, es la herramienta principal del profesor, ya que es la concreción de su trabajo en el aula (lo que enseña y cómo lo hace), mediada por su visión del objetivo de este proceso (por qué y para qué lo hace), es decir, corresponde al conjunto de decisiones explícitas que toma un docente para enseñar un contenido (Couso 2012). Por otra parte, uno de los temas más complejos de comprender en Biología es la teoría de la evolución (Kampourakis 2014), por lo tanto, generar una SEA en este tema puede ser un gran aporte para el profesorado y la investigación en el ámbito de la enseñanza y aprendizaje de la evolución. En esta SEA, las actividades fueron diseñadas según su finalidad didáctica, diferenciadas entre actividades de exploración, introducción, estructuración y aplicación (Sanmartí 2002).

De esta forma, el estudio tuvo como objetivo general diseñar e implementar una SEA para el aprendizaje de la evolución en estudiantes de octavo año de educación básica (13 años, $2^{\circ}$ ESO), en la cual se incluyen aspectos de NdC. Desde este objetivo y tras la revisión bibliográfico, se generó la siguiente pregunta que guío la investigación: ¿Cómo impacta la apropiación de los aspectos de $\mathrm{NdC}$ en la comprensión y aceptación de la evolución? A partir de esta pregunta, se generó la siguiente hipótesis: la mayor comprensión de aspectos propios de $\mathrm{NdC}$ contribuye a una mayor comprensión y aceptación de la teoría de la evolución.

\section{Metodología}

La presente investigación tuvo un enfoque cuantitativo, con un tipo de estudio explicativo y un diseño cuasi experimental. En este diseño, el objetivo es estudiar una situación de la cual no existe un control absoluto de los participantes y al mismo tiempo, la selección de los sujetos participantes no ha sido aleatoria (Fischer, Boone y Neumann 2014). Ante esto, se incluyen grupos establecidos previo a la implementación de la SEA, en este caso 3 cursos de octavo año básico. De esta forma, en uno de los cursos (A) se trabajó de forma explícita la NdC y su conexión con el conocimiento evolutivo. En otro de los cursos (B) se trabajó de forma semi-explícita la $\mathrm{NdC}$, en el cual no se realizaron discusiones guiadas sobre los aspectos, pero si se in- 
cluyeron en los elementos en las actividades, y en un último curso $(\mathrm{C})$, donde no se trataron elementos de la $\mathrm{NdC}$.

\section{Participantes}

La presente investigación se realizó en un colegio privado de Santiago, Chile. El establecimiento cuenta con 3 cursos por nivel con un promedio de 25 estudiantes por curso. Para el cumplimiento del objetivo de esta investigación, se realizó una SEA en octavo año básico $\left(2^{\circ}\right.$ de ESO). La muestra total del estudio estuvo integrada por 48 estudiantes quienes realizaron los pre y postest.

\section{Variables e instrumentos}

Diferentes instrumentos fueron utilizados para operacionalizar las tres variables principales: 1) aceptación de la evolución, 2) comprensión de la evolución y 3) comprensión de la naturaleza de la ciencia. Se utilizaron instrumentos válidos y confiables disponibles en la literatura (Lederman et al. 2002; Rutledge y Warden 2000) para evaluar la aceptación de la evolución y la comprensión de la naturaleza de la ciencia. Para la aceptación, se utilizó el cuestionario MATE (por su nombre en inglés, Measure of Acceptance of the Theory of Evolution MATE) y para la comprensión de $\mathrm{NdC}$, el cuestionario VNOS-D+ (por su nombre en inglés Vision of Nature of Science). Ambos instrumentos ya han sido utilizados y validados en sus versiones en español en estudios anteriores (Cofré, Vergara, Lederman, Lederman, Santibánez, Jiménez y Yancovi 2014; Cofré et al. 2017). En el caso del MATE (Rutledge y Warden 2000), el instrumento corresponde a un cuestionario de escala Likert con 20 preguntas (tabla 1). Este instrumento originalmente fue desarrollado para su uso con profesores de biología, pero se ha utilizado también para analizar la aceptación de estudiantes de secundaria (Cavallo y McCall 2008; Cofré et al. 2017), estudiantes de pedagogía en Biología y estudiantes de nivel superior en cursos de biología evolutiva (Ingram y Nelson 2006). De acuerdo a sus autores, el análisis del cuestionario se realiza al asignar 5 puntos a cada respuesta correcta, por lo que un nivel máximo de aceptación de la evolución implica un puntaje de 100. El nivel mínimo de aceptación serán 20 puntos. Se considera una aceptación alta sobre los 77 puntos (Rutledge y Warden 2000).

Tabla 1. Preguntas cuestionario MATE.

1. La evolución es una teoría científica válida.

2. Los organismos existentes en la actualidad son el resultado de procesos evolutivos que han ocurrido durante millones de años.

3. La teoría de la evolución se basa en la especulación y en observaciones y pruebas científicas no válidas.

4. Los seres humanos modernos son producto del proceso de evolución que ha tenido lugar durante millones de años.

5. Existe un cuerpo considerable de datos que apoyan la teoría de la evolución.

6. La mayoría de los científicos aceptan la teoría de la evolución como una teoría científica válida.

7. La teoría de la evolución es incapaz de ser científicamente comprobada.

8. La teoría de la evolución no puede ser correcta, ya que no está de acuerdo con el relato bíblico de la creación.

9. Con pocas excepciones, los organismos de la Tierra se originaron todos al mismo tiempo

10. La edad de la Tierra es de menos de 20.000 años.

11. La teoría de la evolución da sentido a las diversas características y comportamientos observados en los seres vivos. 
12. La teoría evolutiva genera predicciones comprobables con respecto a las características de la vida

13. Los organismos existentes en la actualidad son, esencialmente, de la misma forma que han sido siempre.

14. La evolución no es una teoría científica válida.

15. Gran parte de la comunidad científica duda de si se produce la evolución.

16. La teoría de la evolución es el resultado de investigación y metodología científica actual.

17. La teoría de la evolución se apoya en datos empíricos, históricos, y de laboratorio.

18. Los seres humanos existen hoy en día de la misma forma en que han existido siempre.

19. La edad de la Tierra es de aproximadamente 4-5 mil millones de años.

20. La evidencia disponible es ambigua en cuanto a si realmente se produce la evolución.

Para el caso de la NdC, el cuestionario VNOS-D+ corresponde a 8 preguntas abiertas, las cuales evalúan la comprensión de 7 aspectos de la NdC (Cofré 2012). Ya que en esta investigación sólo se incluyeron 4 aspectos de la NdC descritos por Lederman et al. (2002), los estudiantes respondieron únicamente las preguntas que hacían referencia a los aspectos trabajados (observación e inferencia; carácter empírico; subjetividad y teoría y ley). Estas preguntas fueron: 1, 3a, 3b, 3c y 6 (ver tabla 2). De acuerdo a la metodología estándar usada para analizar este instrumento, se reconocieron respuestas: informadas, mixtas o ingenuas. La respuesta informada corresponde a una visión claramente alineada con el conocimiento recomendada para cada aspecto de $\mathrm{NdC}$. Cuando la respuesta se alineó con lo recomendado pero no estaba completamente desarrollada y poseía algunos conceptos erróneos, fue clasificado como mixta. Cuando la respuesta correspondía claramente a una concepción sobre qué es la ciencia y cómo se construye el conocimiento científico, se clasificó como ingenua. Con el objetivo de realizar un análisis cuantitativo del conocimiento de $\mathrm{NdC}$, se asignaron valores de 2 puntos a las respuestas informadas, 1 a las mixtas y 0 para las respuestas ingenuas. Los valores de cada aspecto se sumaron para obtener un valor total para el conocimiento de $\mathrm{NdC}$ por estudiante.

Tabla 2. Preguntas del cuestionario VNOS-D+

1. ¿Qué es la ciencia para usted? ¿Que la hace diferente de otras disciplinas como el arte y la religión?

2. Los científicos producen conocimiento científico.¿Usted cree que este conocimiento puede cambiar en el futuro? Explique su respuesta y de un ejemplo.

3. (a) ¿Como saben los científicos que los dinosaurios existieron realmente? (b) ¿Cuán seguros están los científicos del aspecto que tenían los dinosaurios? (c) Los científicos están de acuerdo que cerca de 65 millones de años atrás los dinosaurios se extinguieron (todos los individuos dinosaurios murieron). Sin embargo, los científicos no están de acuerdo sobre cual fue la causa que causo esta extinción masiva. ¿Por qué cree usted que ellos no están de acuerdo aun cuando ellos pueden accede a la misma información?

4. En relación a la predicción del tiempo atmosférico, los meteorólogos recolectan información de diferente tipo. A menudo ellos producen modelos en computador de los diferentes patrones del tiempo. (a) ¿Usted cree que los meteorólogos están seguros sobre el funcionamiento de los modelos computacionales de los patrones del tiempo atmosférico? (b) ¿Por que si o por que no?

5. Una joven investigadora estudió muchos tipos de pájaros. Ella se dio cuenta de que los pájaros que comen los mismos tipos de comida usualmente tiene un pico de la misma forma. Por ejemplo, muchos pájaros que comen nueces tienen un pico fuerte y corto, y muchos pájaros que comen gusanos que extraen de la superficie de la tierra, tienen picos largos y delgados. De manera que la joven decidió que había una conexión entre la forma del pico y el tipo de comida que comen los pájaros.

6. Los científicos tratan de encontrar respuestas a sus preguntas haciendo investigaciones/experimentos. ¿Usted cree que los científicos utilizan su imaginación y su creatividad cuando ellos investigan/hacen 
experimentos? (a). Si la respuesta es NO, explique por qué, (b). Si la respuesta es Si, ¿̇en que parte(s) de su investigación (diseño, experimentación, hacer observaciones, análisis de datos, interpretación, exposición de resultados, etc.) usted cree que ellos usan la imaginación y la creatividad?. De ejemplos si puede

7. ¿Existen diferencias entre teorías científicas y leyes científicas? Explique las diferencias e ilustre con un ejemplo.

8. Algunos proponen que la ciencia es permeada con valores sociales y culturales. Esto quiere decir que la ciencia refleja valores políticos y sociales, creencias filosóficas y normas intelectuales de la cultura en la cual esta es practicada. Otros proponen que la ciencia es universal. Esto es, la ciencia trasciende las naciones y los limites culturales y no es afectada por valores filosóficos, políticos o sociales, y por las nor mas intelectuales de la cultura donde ésta se practica. 5. ¿Qué es para ti un modelo en ciencias? Describe sus características y funciones 6 . ¿Cómo se define un modelo en ciencias?

Finalmente, para evaluar el conocimiento sobre evolución y tiempo geológico, se generó un instrumento nuevo el cual fue validado. El instrumento consta de 12 preguntas de selección única (Tabla 3) con 4 alternativas, las cuales fueron creadas por las autoras del estudio en directa relación con los temas revisados en el currículo de octavo año de enseñanza básica para el tema de evolución.

Tabla 3. Ejemplo de pregunta

P1. El picaflor de la isla de Juan Fernández tiene, en la actualidad, muy pocos representantes vivos. ¿Cómo se denominaría la especie si mueren todos sus miembros? a) Preservada, b) Extinta, c) Fosilizada, d) Amenazada

Estas preguntas fueron revisadas por dos expertos en contenido evolutivo y un experto en diseño de instrumentos. Tras la validación por expertos en términos de contenido y constructo, se realizó un pilotaje de todos los instrumentos con un grupo pequeño de 6 estudiantes, para revisar temas de redacción y compresión de instrucciones. Para el análisis de esta variables, se asignó 1 punto a cada pregunta correctamente respondida, mientras las incorrectas tienen valoración 0.

\section{Creación, validación e implementación de la Secuencia}

La progresión de las actividades fue desde el surgimiento progresivo de formas de vida a través del tiempo geológico, desde sus las primeras manifestaciones de la vida, enfatizando la evolución como un proceso de cambio. Inicia con un trabajo grupal e individual sobre el dibujo de un mamífero de Chile y su ancestro, posteriormente, se trabaja con láminas sobre mamíferos extintos de Chile y se organizaron en el tiempo en base a las eras geológicas. A continuación, se les realizaron varias cuestiones en base al trabajo realizado, para que los estudiantes lograran exponer y comentar la experiencia del trabajo con SEA en equipos. Finalmente, se observando de manera general un cortometraje titulado The day be died the Mesozoic (El día en que murió el Mesozoico) divulgado por HHMI Biointeractive. El objetivo de este material es revisar hipótesis relacionadas con la extinción de los dinosaurios. Para ello, se describen varias posturas de científicos de distintas áreas, por lo que se hace propicio para evidenciar diversos aspectos de $\mathrm{NdC}$ que ya se habían trabajado durante la implementación de la SEA. Además de visualizar los cambios de las especies a través del tiempo, se busca que los estudiantes logren comprender empíricamente en una discusión científica real algunos aspectos de NdC. En esta SEA, en cada etapa se les preguntaba al finalizar las sesiones de clases por medio de un ticket de salida, cuáles son las ideas clave de la actividad realizada, qué es lo que habían comprendido y cómo lo iban relacionando con el concepto de evolución. Al término de la SEA, los estudiantes contestaron la co y autoevaluación, con lo que se 
completaba el proceso evaluativo. Lo relevante es que son algunos aspectos para enseñar evolución y $\mathrm{NdC}$, como, por ejemplo: observación e inferencia, teoría y ley, subjetividad y carácter empírico.

Para validar el diseño de la secuencia, recurrimos a un esquema mixto que hemos denominado interno-externo. La validación interna se llevó a cabo mediante el ciclo de Jhon Elliot (1993) de investigación-acción, la cual se implementó en un grupo pequeño para revisar y corregir el material confeccionado en cuanto a su nivel de complejidad, calidad de instrucciones, redacción, entre otros. En cuento a la validación externa, recurrimos al modelo de evaluación respondiente de Robert Stake (1967), comparando el material diseñado con otros productos de circulación nacional.

Tabla 4. Descripción general de las actividades incluidas en la SEA.

\begin{tabular}{|c|c|c|}
\hline Tipo de actividad & Momento de SEA & Descripción general de las actividades \\
\hline \multirow[t]{4}{*}{ Exploración } & \multirow{4}{*}{$\begin{array}{l}\text { Sesión } 1 . \\
\text { Diversidad de } \\
\text { mamíferos } \\
\text { extintos en Chile }\end{array}$} & $\begin{array}{l}1 \text { Ítem: A partir del dibujo de un mamífero a elección se inda- } \\
\text { ga en las concepciones alternativas en relación a evolución, } \\
\text { adaptación y ancestro común. }\end{array}$ \\
\hline & & $\begin{array}{l}2 \text { Ítem: A partir del ítem } 1 \text { se discute en equipo concepciones } \\
\text { alternativas compartidas y diferenciadas. }\end{array}$ \\
\hline & & $\begin{array}{l}\text { Glosario NdC: Se describe qué es en Ciencia un dato y el tra- } \\
\text { bajo en equipo. }\end{array}$ \\
\hline & & Ticket de Salida \\
\hline \multirow[t]{2}{*}{$\begin{array}{l}\text { Introducción de } \\
\text { Nuevos } \\
\text { conocimientos }\end{array}$} & \multirow[t]{2}{*}{$\begin{array}{c}\text { Sesión } 2 . \\
\text { Situemos a los } \\
\text { mamíferos en el } \\
\text { tiempo }\end{array}$} & $\begin{array}{l}\text { Ítem 1: Análisis de un modelo explicativo de tiempo geológi- } \\
\text { co. } \\
\text { Trabajo con nueva información: láminas de mamíferos extin- } \\
\text { tos de Chile. } \\
\text { Comprensión de aspectos taxonómicos entregados en las lá- } \\
\text { minas. } \\
\text { Reorganización de la información expuesta en las láminas. }\end{array}$ \\
\hline & & Ticket de Salida \\
\hline \multirow[t]{3}{*}{ Estructuración } & \multirow{3}{*}{$\begin{array}{l}\text { Sesión } 3 . \\
\text { Recordemos lo } \\
\text { trabajado en } \\
\text { clases }\end{array}$} & $\begin{array}{l}\text { Ítem 1: Comprensión y análisis de nuevos conocimientos rela- } \\
\text { cionadas con periodos, orden, mamíferos, evolución y tiempo } \\
\text { geológico. }\end{array}$ \\
\hline & & Glosario: observación e inferencia, fidedigno y dato empírico. \\
\hline & & Ticket de Salida \\
\hline \multirow[t]{4}{*}{ Aplicación } & \multirow[t]{2}{*}{$\begin{array}{l}\text { Sesión } 4 . \\
\text { Apliquemos lo } \\
\text { aprendido }\end{array}$} & $\begin{array}{l}\text { Ítem 1: Análisis guiado de video "El día que murió el Meso- } \\
\text { zoico", de manera paralela van registrando hipótesis, datos y } \\
\text { evidencias que entrega el documental sobre la posible extin- } \\
\text { ción de los dinosaurios. }\end{array}$ \\
\hline & & Ticket de Salida \\
\hline & \multirow{2}{*}{$\begin{array}{l}\text { Sesión } 5 \\
\text { Apliquemos lo } \\
\text { aprendido }\end{array}$} & $\begin{array}{l}\text { Registran a modo de síntesis e integración de nuevos conteni- } \\
\text { dos conocimientos alcanzados por medio de la SEA. }\end{array}$ \\
\hline & & Ticket de salida. \\
\hline
\end{tabular}




\section{Análisis de los datos}

Para poder evaluar en términos cuantitativos el cambio (o no) en la comprensión y aceptación de evolución de los estudiantes, así como su visión de $\mathrm{NdC}$, se realizaron dos pruebas estadísticas. Cuando los datos se distribuyeron de forma normal, se realizó el test de Students para muestras pareadas y cuando los datos no cumplieron este supuesto, se realizó el test no paramétrico de comparaciones de medias relacionadas Wilcoxon Signed-Rank (Fiel, 2009).

\section{Resultados}

\section{Comprensión de la $\mathrm{NdC}$}

De acuerdo al análisis estadístico realizado, se pudo constatar que la intervención de la SEA que consideró aspectos de $\mathrm{NdC}$ de forma explícita (grupo A) mejoró significativamente la visión del quehacer científico de los estudiantes. Según el test de Wilcoxon signed-rank, la comprensión de $\mathrm{NdC}$ antes de la secuencia fue significativamente menor a la comprensión tras las clases ( $\mathrm{z}=-3.2 ; \mathrm{p}<0,01)$ en el curso con $\mathrm{NdC}$ explícito. Sin embargo, en los otros dos cursos, donde no se trató el tema de $\mathrm{NdC}$ de forma explícita, antes o durante las clases de evolución, la comprensión de $\mathrm{NdC}$ de los estudiantes previa a las clases no fue distinta a la registrada al final de las clases. Para curso B con NdC semi-explícito ( $\mathrm{z}=-0.81 ; \mathrm{p}=0,168)$ y el curso $\mathrm{C}$ grupo control $\sin \mathrm{NdC}(\mathrm{z}=0.2 ; \mathrm{p}=0,589)$. Específicamente, los aspectos que más mejoraron en el curso de $\mathrm{NdC}$ explícito fueron la comprensión de la diferencia entre observación e inferencia y la diferencia entre teoría y ley.

\section{Aceptación de Teoría de la Evolución}

En cuanto a la aceptación de la evolución, en general los tres cursos presentan niveles altos de aceptación desde el comienzo; valor comprendido entre 74 y 80 (Figura 1). En la figura, también se puede observar que tras la secuencia todos los cursos aumentan en promedio su aceptación de la teoría de la evolución. Sin embargo, solo algunos de estos aumentos son estadísticamente significativos. Para el grupo A (NdC explícito), el test de Students mostró una diferencia significativa entre la media inicial $(M=76,53, S E=1,76)$ y la final $(M=81,13, S E=1,79)$. $(\mathrm{t}(15)=-2.4 ; \mathrm{p}<0,05, \mathrm{r}=0,58)$. Al observar los resultados del grupo $\mathrm{B}$ ( $\mathrm{NdC}$ semi explícito), la aceptación promedio antes de la SEA $(\mathrm{M}=80,21, \mathrm{SE}=2,86)$ no fue estadísticamente menor a la aceptación promedio después de la SEA ( $\mathrm{M}=81,78$, SE=2,96) $(\mathrm{t}(14)=-0.9 \mathrm{p}>0,2, \mathrm{r}=0,09)$. Finalmente, los resultados del grupo $\mathrm{C}$ (Grupo control sin $\mathrm{NdC}$ ) también son estadísticamente significativos, es decir la aceptación inicial $(\mathrm{M}=74,47, \mathrm{SE}=2,28)$ es menor a la final $(\mathrm{M}=77,26$, $\mathrm{SE}=2,14)(\mathrm{t}(19)=-2.8, \mathrm{p}<0,05, \mathrm{r}=0,82)$. 


\section{Resultados MATE}

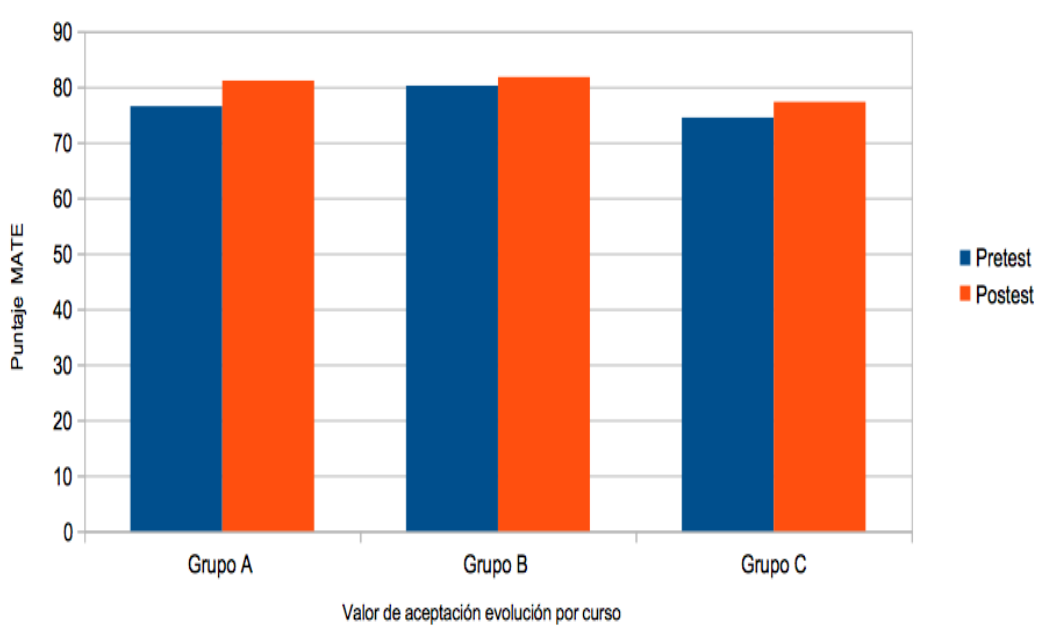

Figura 1: Resultados cuestionario MATE por curso. En este test el puntaje máximo es 100 puntos. Las barras azules muestran el valor del pretest y la barra naranjas el valor en el postest.

\section{Comprensión de Teoría de la Evolución}

En relación a la comprensión de la evolución y el tiempo geológico, al comienzo de la SEA se observa que dos de los tres cursos obtiene un promedio insuficiente (menor a 7 respuestas correctas equivalente a un logro inferior al 60\%) (Figura 2). Una vez realizada la SEA, se puede observar que los tres cursos mejoran su comprensión de la evolución. El grupo A logra un promedio de 9,6 respuestas correctas, el grupo B logra un promedio de 10,3 respuestas correctas y el grupo C logra un promedio de 10,1 respuestas correctas. Estos aumentos son en los tres casos estadísticamente significativos de acuerdo al test de Wilcoxon signed-rank (grupo A: $z=-2,8 ; p<0,01$; grupo $B: z=-3,1 ; p<0,01$ y grupo $C: z=4,1 ; p<0,001$ ).

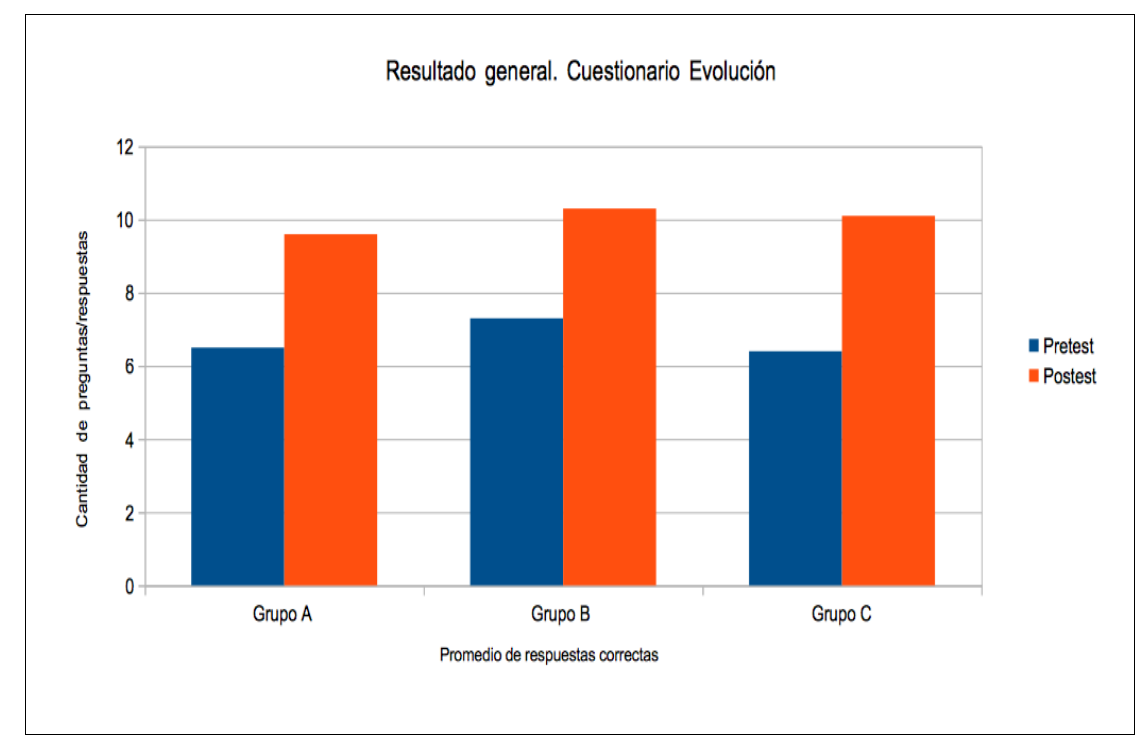

Figura 2: Promedios de respuesta por curso. 


\section{Discusiones}

En cuanto a la aceptación de evolución (MATE, figura 1), se observa que el grupo que trabajó $\mathrm{NdC}$ explícitamente logra comparativamente un aumento en relación a su pre y postest vs. los otros cursos participantes en la investigación. En general los resultados apuntan a que la comprensión de los aspectos de $\mathrm{NdC}$ se alcanza mayormente cuando el trabajo es formalmente planificado dentro de las clases. Se muestra que cuando se trabaja $\mathrm{NdC}$ de manera cuasi explicita se logra aumentar la comprensión de los aspectos, pero en menor grado (Grupo B). Cuando los aspectos de $\mathrm{NdC}$ no son trabajados explícitamente ni cuasi explícitamente (Grupo C), no se logra visualizar ninguna comparación significativa entre los aspectos de $\mathrm{NdC}$ propios de esta investigación. Por lo tanto, ante estos datos podemos decir que la comprensión de $\mathrm{NdC}$ solo se alcanza cuando existe un trabajo planificado y no es inherente a la comprensión de conocimiento de evolución. En cuanto al conocimiento disciplinar propio de la evolución (figura 2), se observa que tanto los cursos que trabajaron con $\mathrm{NdC}$ explícitamente como cuasi explícitamente logran menor avance que el curso que sólo se enfocó en trabajar evolución sin destinar tiempo ni actividades de NdC. Entonces, ¿cómo impacta la comprensión de los aspectos de $\mathrm{NdC}$ en el aprendizaje de evolución en estudiantes?

\section{Naturaleza de la Ciencia, Comprensión y Aceptación de la Evolución}

Según los resultados obtenidos en este trabajo, podemos observar que los aspectos de NdC que fueron trabajados (observación e inferencia, carácter empírico, subjetividad, teoría y Ley), tuvieron una relación causal con los resultados del MATE (figura 1) obtenidos en el grupo A, ya que este curso donde se aplicó $\mathrm{NdC}$ de forma explícita, tuvo un aumento significativo en los aspectos de $\mathrm{NdC}$ trabajados, donde los resultados de los estudiantes avanzaron del nivel ingenuo al mixto e informado y, a su vez, hubo un aumento en los resultados de la aceptación de la evolución. Esto nos permitió probar que se hace más significativo el aprendizaje si se trabajan aspectos de $\mathrm{NdC}$ para la comprensión de evolución en el aula; ello, no ocurrió con el $\mathrm{B}$ y $\mathrm{C}$, donde el aumento no fue significativo, lo que se atribuyó al tratamiento de clases planificadas; en ellas no existía trabajo de $\mathrm{NdC}$ explícito.

A pesar de que existen poco estudios sobre la relación significativa entre $\mathrm{NdC}$ y la aceptación de la evolución, como lo señala la revisión reciente de Glaze y Goldston (2015) y Rivas y González García (2016), no se debe dejar pasar esta oportunidad para hacer énfasis en la relevancia de la reflexión de los aspectos de $\mathrm{NdC}$ en el aula. Permite que los estudiantes consideren la evolución como un conocimiento científico válido y toman conciencia de que el trabajo de la Ciencia va más allá de observar o trabajar en el laboratorio. Por tanto, toma importancia la formación científica de nuestros estudiantes (Akyol et al. 2012).

Por otra parte, es importante detenerse en los resultados de aspectos de NdC (VNOS-D) de los pretest que se aplicaron en los tres cursos estudiados. Los estudiantes se encontraban principalmente en el nivel ingenuo y mixto, lo que concuerda con las investigaciones realizadas por diversos autores (Lederman et al. 2002; Cofré 2012). Es importante destacar que los estudiantes que trabajaron con la SEA, donde se incluía algunos aspectos de $\mathrm{NdC}$, tuvieron un avance desde el nivel ingenuo al mixto y del mixto al informado, siendo el grupo A quien tuvo el mayor avance por realizar más reflexiones en el aula con sus estudiantes, lo que permitió una mejor asimilación de los conceptos tratados. Se debe destacar al mismo tiempo que tal como se trató en el análisis de resultados, el aspecto de teoría y ley fue el que menos tuvo resultados significativos, ya que solo se trabajó una pregunta en la SEA y no se abordó con 
mayor profundidad este aspecto. Por ejemplo, ante la pregunta: $\dot{i}$ Existen diferencias entre teorias cientificas y leyes científicas? Explique las diferencias e ilustre con un ejemplo:

[...] si, porque las teorías tienen más investigaciones ya que todavia no son oficiales, en cambio las leyes no cambian por eso son leyes. Por ejemplo, en la teoría del big bang todavía sigue saliendo información. Y en la ley de obm ya no bay mas información porque es asíy no va a cambiar.[...] (A14).

\section{SEA como recurso para enseñar Evolución}

Como se planteó al principio de este trabajo, esta SEA tuvo por objetivo que los estudiantes de 13 años aprendieran evolución, lo que conllevó dos grandes actividades que permitieron a los estudiantes comprender de mejor manera lo que significa evolución en nuestros días. Esto se vio reflejado en los resultados del cuestionario sobre conocimientos de evolución, ya que según lo que observa en la figura 2 , hubo un aumento en el conocimiento de este contenido en los tres cursos, siendo el grupo $\mathrm{C}$ el que tuvo un mayor aumento debido a que su SEA no tenía aspectos de NdC por lo que se enfocó 100\% en reflexiones en base solo al contenido tratado. Esto es importante destacarlo, ya que este resultado nos deja ver que la SEA realizada cumplió con los objetivos planteados inicialmente en esta investigación, lo que nos recalca la importancia de utilizar buenos recursos en clases o nuevas metodologías para enseñar un contenido. Estos resultados coinciden con lo descrito en la literatura, donde se señala que clases no tradicionales centradas en el estudiante, como lo fue en este caso la SEA, pueden mejorar el conocimiento de ellos sobre la evolución (Glaze y Goldston 2015).

\section{Conclusiones e implicancias para el aula}

Una conclusión que se levanta a partir de los resultados de los test aplicados, es que NdC sólo es aprendida cuando existe una planificación estructurada para su enseñanza y no es un aprendizaje inherente a la comprensión de los contenidos de Ciencia. Incluso, la comparación entre el pre y postest VNOS-D muestra que en algunos aspectos la comprensión de NoS bajó posterior a la implementación de la SEA, (grupo C, grupo control donde no se trabajó $\mathrm{NdC}$ ). Es el caso del aspecto "Teoría y Ley", antes de la implementación de la SEA 13 estudiantes tenían una comprensión ingenua y al finalizar la SEA, esta comprensión había subido a 15 estudiantes. Estos resultados se relacionan con investigaciones ofrecidas en la literatura actual (Glaze y Goldston 2015; Nadelson y Hardy 2015).

Es importante destacar que los estudiantes de octavo año básico lograron una comprensión general de evolución sin estudiar los mecanismos evolutivos, tan solo se dedicaron a discutir sobre datos y evidencias generales como registro fósil o catástrofes que cambiaron las condiciones ambientales del planeta. Desde aquí, se trabaja y potencia la idea de que para enseñar Ciencia y principalmente características propias de la Ciencia, no es necesario contar con un ambicioso programa curricular o muchos objetivos y contenidos de complejo aprendizaje. La manera en la que se enseñó Ciencia en esta SEA fue propiciando momentos de discusión en equipo, permitiendo a los estudiantes revisar diferentes fuentes de información, cuestionarse en equipos y desde esta modalidad los mismos estudiantes notaban los aspectos propios de la Ciencia que se volvían poco a poco más evidentes. Claramente, para lograr este propósito es necesario que las planificaciones de clase sean lo más reflexionadas posibles, no basta con ceñirse a un ciclo de aprendizaje, es primordial que un docente logre vislumbrar anticipadamente posibles preguntas, inquietudes, concepciones alternativas que se levantaran y posiblemente arrastrar (erróneamente o no). Desde aquí, se sostiene que toda intervención debe contar con un cierre que permita dar evidencias, por ejemplo, un diálogo dirigido, preguntas guiadas, ticket de salida, debates breves, etc. 
Por lo tanto, podemos afirmar que conseguimos cumplir los objetivos y pregunta planteada en esta investigación, logrando visualizar que la implementación de una SEA donde se incluían actividades individuales y grupales permitían generar debate y reflexiones sobre lo que se iba aprendiendo de evolución. Esto fue muy significativo para los estudiantes, sobre todo al incluir aspectos de $\mathrm{NdC}$, que permitieron generar una mayor aceptación de este contenido por parte de los estudiantes de octavo básico.

Finalmente, destacar el hecho de que los aspectos de $\mathrm{NdC}$ trabajados en esta SEA son curriculares y pese a ello, el nivel de comprensión alcanzado por los estudiantes en las clases formales de Ciencia sólo se alcanzan si este trabajo es planificado e intencionado paralelamente con los contenidos propios de la disciplina. En este caso se utilizó la unidad de Evolución. No podemos definir que sólo se puede aprender $\mathrm{NdC}$ por medio de clases vinculadas con un contenido, posiblemente también se pueda aprender $\mathrm{NdC}$ sin un contexto determinado, pero en ambos casos, la intensión en el trabajo de $\mathrm{NdC}$ es relevante y no es un aprendizaje que llega a los estudiantes solo porque estén aprendiendo contenidos científicos, aprender a pensar científicamente es distinto a aprender contenidos de Ciencia.

\section{Agradecimientos}

Este trabajo fue realizado gracias al apoyo de los proyectos FONDECYT 1150659 y FONDECYT 1131029 de la Comisión Nacional de Ciencia y Tecnología (CONICY'T) Gobierno de Chile.

\section{Bibliografía}

Abell, S. K., Lederman, N. G. (2007) Handbook of Research on Science Education (Vol. 1). Routledge: New York.

Acevedo Díaz, J. A. (2009) Conocimiento didáctico del contenido para la enseñanza de la naturaleza de la ciencia (I): el marco teórico. Revista Eureka sobre Enseñanza y Divulgación de las Ciencias 6(1), 21-46.

Akyol, G., Tekkaya, C., Sungur, S., Traynor, A. (2012) Modeling the interrelationships among pre-service science teachers' understanding and acceptance of evolution, their views on nature of science and self-efficacy beliefs regarding teaching evolution. Journal of Science Teacher Education, 23(8), 937-957.

Banet, E., Ayuso, G. E. (2003) Teaching of biological inheritance and evolution of living beings in secondary school. International Journal of Science Education 25 (3), 373-407.

Bayer, C. N., Luberda, M. (2016) Measure, then show: Grasping human evolution through an inquiry-based, data-driven hominin skulls lab. PLOS ONE, 11(8), e0160054.

Barberá, Ó. (2009). Extinción: una forma distinta de ver la vida. Alambique: Didáctica de las Ciencias Experimentales 15(62), 29-42.

Bardapurkar, A. (2008) Do students see the "selection" in organic evolution?: a critical review of the causal structure of student explanations. Evolution: Education \& Outreach, 1(3), 299-305.

Cañal, P. (2009) Acerca de la enseñanza sobre la evolución biológica en la escuela infantil y primaria. Alambique: Didáctica de las Ciencias Experimentales 15(62), 75-91.

Cavallo AML., McCall D. (2008) Seeing may not mean believing: examining students' understandings \& beliefs in evolution. American Biology Teaching. 70, 522-530.

Clough, M. (2003) Explicit but insufficient: Additional considerations for successful NOS instruction. Trabajo presentado en la Seventh International History, Philosophy and Science Teaching Conference, Winnipeg, Canadá. 
Cofré, H. L. (2012) La Enseñanza de la Naturaleza de la Ciencia en Chile: del Currículo a la sala de clases. Revista Chilena de Educación Cientifica 11(1), 12-21.

Cofré, H. L., Vergara, C. A., Santibáñez, D. P., Jiménez, J. P. (2013) Una primera aproximación a la comprensión que tienen estudiantes universitarios en Chile de la Teoría de la Evolución. Estudios pedagógicos 39(2), 67-83.

Cofré, H. L., Vergara, C., Lederman, N. G., Lederman, J. S., Santibáñez, D., Jiménez, J., Yancovic, M. (2014) Improving Chilean In-service Elementary Teachers' Understanding of Nature of Science Using Self-contained NOS and ContentEmbedded Mini-Courses. Journal of Science Teacher Education 25(7), 759-783.

Cofré, H., Santibáñez, D., Jiménez, J., Spotorno, A., Carmona, F., Navarrete, K., Vergara, C. (2017) The effect of teaching the nature of science on students' acceptance and understanding of evolution: Myth or reality? Journal of Biological Education. doi:10.1080/00219266.2017.1326968

Couso, D. (2012) Las Secuencias didácticas en la enseñanza y el aprendizaje de las ciencias: modelos para su diseño y validación. En A. Camaño (Coord.) Didáctica de la física y la quimica (pp. 57-84). Graó: Barcelona.

Chaves Mejía, G. A. (2010) La teleología como un obstáculo epistemológico en la enseñanza de la evolución. Universidad Nacional de Colombia.

Dagher, Z. R., S. Boujaoude. (2005) Students' Perceptions of the Nature of Evolutionary Theory. Science Education 89, 378-391.

Dobzhansky, T. (1973) Nothing in biology makes sense except in the light of evolution. American Biology Teacher 35, 125-129.

Elliott, J. (1993) El cambio educativo desde la investigación-acción. Ediciones Morata.

Field, A. (2009) Descubriendo la estadística usando SPSS-2. Bookman.

Fischer, H., Boone, W., Neumann, K. (2014) Quantitative Research Designs and Approaches. En: Handbook of Research on Science Education, 2, 18. (p 28)

Furió, C., Vilches, A. (1997) Las actitudes del alumnado hacia las ciencias y las relaciones ciencia, tecnología y sociedad, en Luis del Carmen (coord.). La enseñanza y el aprendizaje de las ciencias de la naturaleza en la educación secundaria. Barcelona: Horsori.

Galli, L. G., Meinardi, E. (2015) Obstacles for the learning of the model of evolution by natural selection in students of secondary school in Argentina. Ciência \& Educação 21(1), 101-122.

Glaze, A. L., Goldston, M. J. (2015) US Science Teaching and Learning of Evolution: A Critical Review of the Literature 2000-2014. Science Education 99(3), 500-518.

Guillen, F. C. (1997). Algunos aspectos a considerar en la enseñanza de la biología. Alba, A. et al. Contenidos relevantes deficiencias naturales para la educación básica. SNTE, México, 53-64.

Ingram EL., Nelson C.E. (2006) Relationship between achievement and students' acceptance of evolution or creation in an upper-level evolution course. Journal of Reserch in Science Teaching 43, 7-24.

Jiménez Aleixandre, M. (1991). Cambiando las ideas sobre el cambio biológico. Enseñanza de las Ciencias. 9, 3, 248-256. 
Kampourakis, K. (2014) Understanding evolution. Cambridge University Press.

Kampourakis, K. (2016) The "general aspects" conceptualization as a pragmatic and effective means to introducing students to nature of science. Journal of Research in Science Teaching. 53, 5, 667-682.

Kampourakis, K., Zogza, V. (2008) Students' intuitive explanations of the causes of homologies and adaptations. Science \& Education 17(1), 27-47.

Kim, S. Y., Nehm, R. H. (2010) A cross-cultural comparison of Korean and American science teachers' views of evolution and the nature of science. International Journal of Science Education 10,1-31.

Lederman, N. G., Abd-el-Khalick, F., Bell, R. L., Schwartz, R. (2002) Views of nature of science questionnaire (VNOS): Toward valid and meaningful assessment of learners' conceptions of nature of science. Journal of Research in Science Teaching 39, 497-521.

Lombrozo, T., A. Thanukos, Weisberg, M. (2008) The importance ofunderstanding the nature of science for accepting evolution. Evolution: Education and Outreach 1, 290-298.

McComas, W. (1998) The nature of science in science education. Rationales and strategies. Dordrecht: Kluwer.

MINEDUC (1998). Marco curricular de la educación media. Objetivos fundamentales y contenidos mínimos obligatorios de la educación media. Santiago de Chile: Ministerio de Educación.

MINEDUC (2009) Ajuste curricular en Chile. Unidad de currículum y evaluación. Santiago de Chile: Ministerio de Educación.

MINEDUC (2012) Programa de Estudio Ciencia Naturales Octavo Año Básico.

MINEDUC (2013) Módulos didácticos Ciencias Naturales.

MINEDUC (2013) Bases Curriculares en Ciencias Naturales de $7^{\circ}$ básico a 2 Medio.

Passmore, C., Stewart, J. A. (2002) modeling approach to teaching evolutionary biology in high schools. Journal of Research in Science Teaching, 39(3)185-204.

Puig, B., Jiménez Aleixandre, M. P. (2009) ¿Qué considera el alumnado que son pruebas de la evolución? Alambique: Didáctica de las Ciencias Experimentales 15(62), 43-50.

Rivas, M. L., González García, F. (2016) ¿Comprenden y aceptan los estudiantes la evolución? Un estudio en bachillerato y universidad. Revista Eureka sobre Enseñanza y Divulgación de las Ciencias 13 (2), 248-263.

Rutledge, M.L., Warden, M.A. (2000) Evolutionary theory, the nature of science \& high school biology teachers: Critical relationships. The American Biology Teacher 62, 23-31.

Sanmartí, N. (2009) Didáctica de las ciencias en la educación secundaria obligatoria. DCE. Síntesis: Madrid.

Stake, R. (1967) The countenance of educational evaluation. Department for Exceptional Children, Gifted Children Section. 